\title{
Regulation of HMGB1 release by inflammasomes
}

\author{
Ben $\mathrm{Lu}^{1,2}$, Haichao $\mathrm{Wang}^{3}$, Ulf Andersson ${ }^{4}$, Kevin J. Tracey ${ }^{1 凶}$ \\ ${ }^{1}$ Laboratory of Biomedical Science, Feinstein Institute for Medical Research, 350 Community Drive, Manhasset, NY 11030, \\ USA \\ 2 The Elmezzi Graduate School of Molecular Medicine, North Shore-LIJ Health system, 350 Community Drive, Manhasset, \\ NY 11030, USA \\ ${ }^{3}$ Department of Emergency Medicine, North Shore University Hospital, Manhasset, NY 11030, USA \\ ${ }^{4}$ Department of Women's and Children's Health, Karolinska Institutet, S-17677 Stockholm, Sweden \\ $\triangle$ Correspondence: kjtracey@nshs.edu
}

Received November 27, 2012 Accepted December 12, 2012

\section{ABSTRACT}

High mobility group box 1 (HMGB1) is an evolutionarily conserved non-histone chromatin-binding protein. During infection or injury, activated immune cells and damaged cells release HMGB1 into the extracellular space, where HMGB1 functions as a proinflammatory mediator and contributes importantly to the pathogenesis of inflammatory diseases. Recent studies reveal that inflammasomes, intracellular protein complexes, critically regulate HMGB1 release from activated immune cells in response to a variety of exogenous and endogenous danger signals. Double stranded RNA dependent kinase (PKR), an intracellular danger-sensing molecule, physically interacts with inflammasome components and is important for inflammasome activation and HMGB1 release. Together, these studies not only unravel novel mechanisms of HMGB1 release during inflammation, but also provide potential therapeutic targets to treat HMGB1-related inflammatory diseases.

\section{KEYWORDS HMGB1, inflammasome, PKR}

\section{THE FUNCTION AND RELEASE OF HMGB1}

High mobility group box 1 (HMGB1), identified more than 30 years ago, is a $25-\mathrm{kDa}$ evolutionarily conserved and ubiquitously expressed protein. The amino sequence of HMGB1 is $98.5 \%$ identical in mammals. The major structural features of HMGB1 are its two DNA-binding domains, termed $A$ and $B$ box, and a negatively charged $\mathrm{C}$-terminal acidic region. HMGB1 contains two nuclear-localization sequences. Most HMGB1 resides in the nucleus and functions as a non-histone chromatin-binding protein (Müller S, et al., 2001). Early work demonstrated that HMGB1 stabilizes chromatin structure and modulates gene transcription by bending the DNA helical structure (Javaherian et al., 1978). Another pioneering study showed that HMGB1 can be localized to cytoplasm, implicating that HMGB1 might also have important functions outside the nucleus (Bustin and Neihart, 1979).

Twenty years later, extracellular HMGB1 was identified as a proinflammatory cytokine and a late mediator of sepsis (Wang et al., 1999). Unlike most classical proinflammatory cytokines (e.g. TNF and IL-1 3 ) which are released early in systemic inflammatory responses, HMGB1 is released in a delayed manner and maintains high level in the circulation after the onset of sepsis (Wang et al., 1999). Extracellular HMGB1 initiates inflammatory responses by signaling through Toll-like receptor 4 (TLR4) (Yang et al., 2010), and exerts chemoattractant activity by interacting with the receptor for advanced glycation end-products (RAGE) (Manfredi et al., 2008; Schiraldi et al., 2012). In this context, fully reduced HMGB1 forms a complex with CXCL12 and greatly promotes migration of immune cells via CXCR4; whereas partially oxidized HMGB1 with a disulfide bond between cysteine 23 and cysteine 45 triggers inflammatory response via TLR4 (Venereau et al., 2012; Schiraldi et al., 2012). Importantly, polyclonal or monoclonal antibodies that neutralize the proinflammatory activity of HMGB1 significantly improve survival during lethal endotoxemia or experimental sepsis (Andersson and Tracey, 2011). Selectively targeting HMGB1 with neutralizing antibodies also establishes that HMGB1 contributes importantly to a number of other disease syndromes, such as rheumatoid arthritis, colitis, seizures, and liver ischemia / reperfusion injury (Andersson and Tracey, 2011; Maroso and et al., 2010). These observations position 
HMGB1 at the intersection between sterile injury and infectious inflammation.

During tissue injury, HMGB1 can be passively released by damaged or necrotic cells. Cells undergoing apoptosis do not release HMGB1 at the early stage. In contrast to apoptotic cells, necrotic cells rapidly release HMGB1 and initiate sterile inflammation in an HMGB1-dependent manner (Scaffidi et al., 2002). During infection-induced inflammation, immune cells activated by exposure to the products of pathogenic bacteria and viruses actively release HMGB1 into the extracellular milieu (Andersson and Tracey, 2011). Notably, extracellular ATP, a typical damage-associate molecule pattern that released by damaged or dead cells, also induces robust HMGB1 release from macrophages, suggesting that immune cells can actively release HMGB1 during sterile injury (Lamkanfi et al., 2010). Together, these findings indicate the importance to an mechanistic understanding of HMGB1 release from activated immune cells in the inflammatory diseases.

\section{REGULATION OF HMGB1 RELEASE BY INFLAMMASOME}

Early observations indicated that pharmacological inhibition of caspase activity significantly reduces HMGB1 release during endotoxemia and experimental sepsis, and significantly improves the survival of the animal. In vitro, non-specific caspase inhibitors block endotoxin-induced HMGB1 release from isolated macrophages. These results suggest that immune cells actively release HMGB1 in a caspases-dependent manner during inflammation (Qin et al., 2006).

Caspases are a group of evolutionarily conserved aspartate-specific cysteine proteases that cleave substrates in the onset of programmed cell death. Notably, programmed cell death is highly linked with immune defenses against pathogen infection throughout species. In highly developed organisms, the inflammasome, an intracellular multi-protein complex, mediates caspase- 1 activation, which in turn induces the maturation of pro-IL-1 $\beta$ and pro-IL-18, and initiates the onset of pyroptosis, a proinflammatory form of programmed cell death (Franchi et al., 2012; Strowig et al., 2012). The inflammasome also has a role as a sentinel complex in the surveillance of the intracellular environment. It consists of at least two distinct components, including pro-caspase-1, and a NOD-Like receptor (NLR) molecule or a molecule of PYHIN family (Rathinam et al., 2012). NLR molecules contain a leucine-rich-repeat (LRR) domain, which was previously thought to exert ligand-binding function. However, the molecular basis of how individual NLR molecules directly recognize their specific ligands is still not fully understood (Rathinam et al., 2012). PYHIN molecules, such as the absent in melanoma 2 (AIM2), are true intracellular receptors, which specifically recognize double-stranded DNA (Rathinam et al., 2012). Most inflammasome protein complexes contain an adaptor protein, termed apoptosis-associated speck-like protein containing a CARD (ASC), which links pro-caspase-1 to the NLR or PYHIN molecules (Strowig et al., 2012). Human and mouse genome encode more than 20 NLR molecules and several distinct PYHIN molecules. Among these molecules, NLRP1, NLRP3, NLRC4, and AIM2, are well-characterized inflammasome components and have been shown to assemble into functional, caspase-cleaving complexes (Franchi et al., 2012; Rathinam et al., 2012; Strowig et al., 2012).

Recent studies reveal that the inflammasome mediates HMGB1 release from activated immune cells in response to a variety of exogenous and endogenous danger signals (Willingham et al., 2009; Lamkanfi et al., 2010). Importantly, genetic deletion of inflammasome components severely impairs HMGB1 release during endotoxemia or bacteriemia (Willingham et al., 2009; Lamkanfi et al., 2010). These studies clearly indicate that the major source for HMGB1 release in sepsis and endotoxemia is subsequent to inflammasome activation. Notably, Dixit and colleagues recently discovered a noncanonical inflammasome which activates caspase-11, rather than caspase-1. Caspase-11 physically interacts with caspase-1, and contributes importantly to the maturation of proIL-1 $\beta$ and pro-IL-18, upon activation by non-canonical stimuli, such as E.coli bacteria (Kayagaki et al., 2011). Intriguingly, caspase-11 can mediate pyroptosis and HMGB1 release in the absence of caspase-1. Genetic deletion of caspase-11 rather than caspase-1 protects mice from a lethal dose of endotoxin (Kayagaki et al., 2011). In line with this, the loss of both IL-1 $\beta$ and IL-18 fails to protect mice from lethal endotoxin administration; whereas HMGB1 neutralizing antibody dose-dependently promotes survival during lethal endotoxemia (Lamkanfi et al., 2010). These observations highlight the role of inflammasome mediated-HMGB1 release in the pathogenesis of sepsis.

Inflammasomes mediate HMGB1 release by inducing pyroptosis (Lamkanfi et al., 2010; Lu et al., 2012). Previous observations demonstrate that cells undergoing apoptosis conventional programmed cell death, do not release HMGB1 at the early stage (Scaffidi et al., 2002). In contrast to apoptosis, pyroptosis induces rapid and robust HMGB1 release (Lamkanfi et al., 2010). Unlike necrosis, cells that undergo pyroptosis have features of DNA fragmentation, which was previously thought to be a specific signature of apoptosis (Miao et al., 2011). Interestingly, translational modifications of HMGB1 can be a marker for the type of cell death that has occurred. Mass spectrometry analysis reveals that HMGB1 released via pyroptosis is hyperacetylated at the nuclear localization sequences (NLSs); whereas HMGB1 released via freeze/thaw cyclesinduced necrosis contains hypoacetylated NLSs (Lu et al., 2012). Together with the finding that HMGB1 hyperacetylation at NLSs is essential for HMGB1 translocation from nucleus to cytoplasm, which facilitates subsequent HMGB1 release (Bonaldi et al., 2003; Evankovich et al., 2010), these observations suggest that HMGB1 release from inflammasome activation is a highly regulated process. 


\section{THE ROLE OF PKR IN INFLAMMASOME ACTIVATION AND HMGB1 RELEASE}

New evidence links the inflammasome, especially the NLRP3 inflammasome, to the pathogenesis of human diseases such as obesity, type 2 diabetes, cardiovascular diseases, gout, sepsis and colitis (Strowig et al., 2012; Wen et al., 2012). The development of therapeutics targeting the inflammasome and HMGB1 release requires knowledge of specific signaling pathways that regulate inflammasome assembly and activation (Strowig et al., 2012). The best characterized inflammasome is the NLRP3 inflammasome, which is triggered by exogenous and endogenous danger signals, including bacteria, RNA virus, double-stranded RNA (dsRNA), extracellular ATP, monosodium uric (MSU) acid crystal, adjuvant aluminum, and free fatty acid (Franchi et al., 2012; Strowig et al., 2012; Wen et al., 2012).

Double-stranded RNA dependent kinase (PKR) is a 65-kDa intracellular protein, which contains two RNA-binding domains at the $\mathrm{C}$-terminus, and a kinase domain at the $\mathrm{N}$-terminus (Dey et al., 2005). Though it was originally identified as an intracellular dsRNA receptor and a key anti-viral protein, recent studies suggest that it also functions as an intracellular stress sensing molecule. In addition to dsRNA, bacterial components and free fatty acid can induce PKR autophosphorylation (Hsu et al., 2004; Nakamura et al., 2010). In the light of these findings, we recently explored the potential role of PKR in inflammasome activation and HMGB1 release (Lu et al., 2012). Classical inflammasome stimuli, such as extracellular ATP, MSU, and adjuvant aluminum, induce robust PKR autophosphorylation. Genetic deletion or pharmacological inhibition of PKR significantly reduces inflammasome activation induced by various NLRP3 agonists. Overexpression of PKR significantly enhances the activity of the reconstituted NLRP3 inflammasome in non-immune cells. PKR physically interacts with NLRP3. Importantly, PKR autophosphorylation in a cell-free system with recombinant NLRP3, ASC, and pro-caspase-1 reconstitutes inflammasome activity. These findings clearly indicate that PKR can directly regulate the NLRP3 inflammasome activation. Unexpectedly, PKR also physically interacts with other inflammasome components, specifically NLRP1, NLRC4, and AIM2, which broadly regulates the activation of various types of inflammasomes (Lu et al., 2012).

Based on these other findings, we propose that the efficient activation of the inflammasome requires both pattern recognition and stress-sensing (Fig. 1).

\section{Recognition of specific inflammasome ligand}

Intracellular inflammasome ligands initiate oligomerization of the NLR or PYHIN molecules and the assembly of the inflammasome protein complex by directly or indirectly interacting with specific inflammasome components (Franchi et al., 2012; Rathinam et al., 2012). This step is required for the inflammasome activation, and serves as the decision-making mechanism through which the immune system determines which type of inflammasome to be activated in response to a particular

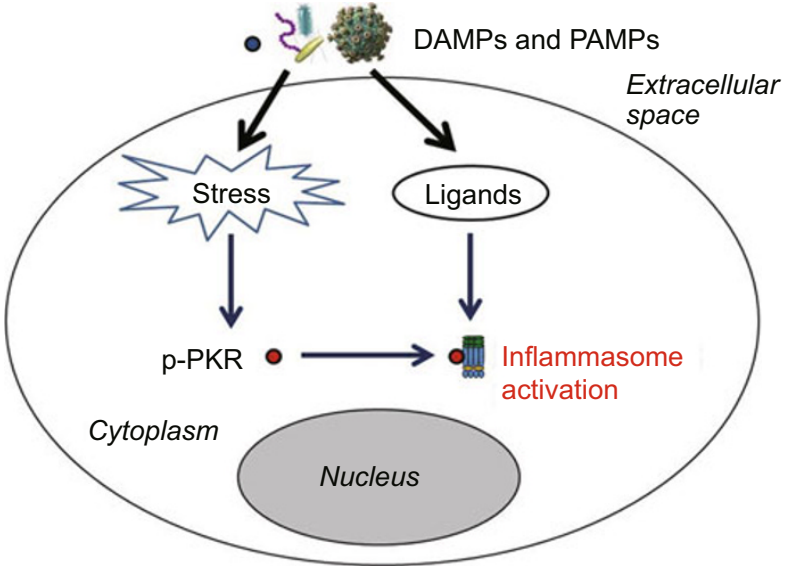

Figure 1. Efficient inflammasome activation requires both pattern recognition and stress sensing. Intracellular inflammasome ligands, derived directly or indirectly from various DAMPs or PAMPs, initiate oligomerization of the NLR or PYHIN molecules and the assembly of the inflammasome protein complex. These DAMPs or PAMPs also induces cellular stress, which in turn mediates PKR autophosphorylation. Phosphorylated PKR functions as a component of inflammasome protein complex and importantly enhances inflammasome activity by facilitating inflammasome assembly.

infectious agent or stimulus. Recent studies have unraveled important molecular mechanisms by which specific inflammasome ligands initiate the assembly and activation of the AIM2 and NLRC4 inflammasomes).

AIM2 consists of a Pyrin domain and a HIM domain. In the absence of ligands, these two domains within AIM2 molecule are in an autoinhibited state. AIM2 is an intracellular DNA receptor that recognizes double-stranded DNA through the HIM domain (Rathinam et al., 2012). The crystal structure of the HIM domain/DNA complex reveals that DNA recognition by AIM2 is mediated by the electrostatic attraction between the positively charged HIM domain residues and the dsDNA sugarphosphate backbone (Jin et al., 2012). Recognition of dsDNA by the HIM domain subsequently liberates the Pyrin domain, which recruits ASC to form an inflammasome protein complex. AIM2 and dsDNA interaction also facilitates inflammasome assembly by recruitment of multiple AIM2 molecules along the DNA staircase (Jin et al., 2012).

The NLRC4 inflammasome is triggered by intracellular bacterial flagellin and the inner rod protein of the type III secretion systems of diverse bacterial species (e.g, PrgJ of Salmonella enterica serovar Typhimurium) (Franchi et al., 2012). The NLRC4 inflammasome recognizes these structurally distinct ligands via a group of NLR families, termed apoptosis inhibitory proteins (NAIPs) (Franchi et al., 2012). Specifically, NAIP5 or NAIP6 complexes with NLRC4 in response to bacterial flagellin; whereas NAIP2 meditates the recognition of PrgJ by NAIP2-NLRC4 complex. These events are followed by the oligomerization of NLRC4 and the assembly of the NLRC4 in- 
flammasome (Kofoed and Vance., 2011; Zhao et al., 2011).

\section{Cellular or environmental stress sensing}

Upon activation in response to cellular or environmental stress, PKR physically interacts with inflammasome components, and is essential for the efficient activation of various inflammasomes (Lu et al., 2012). As inflammation can be considered to be both a tissue stress response and an adaptive response to noxious conditions (Medzhitov, 2010), the recent identification of PKR as a critical regulator of inflammasomes has provided a novel insight into how the immune system amplifies inflammasome activation and adjusts the intensity of inflammation in response to cellular or environmental stress (Lu et al., 2012).

\section{FUTURE PERSPECTIVE}

The majority of HMGB1 is localized in the nucleus of cells in the resting state. Previous studies established that HMGB1 translocation from nucleus to cytoplasm is an essential step in HMGB1 release from activated immune cells. After exposure to the infectious agents or endogenous danger signals, including LPS, bacteria, virus, and extracellular ATP, immune cells mobilize nuclear HMGB1 into cytoplasm (Lu et al., 2012; Andersson and Tracey, 2011; Lamkanfi et al., 2010), where HMGB1 is delivered into a vesicle-like organelle, termed the secretary lysosome, as shown by electron microscope (Gardella et al., 2002). Mechanistic studies reveal that nuclear localization of HMGB1 depends on its two nuclear localization sequences (NLSs). Acetylation of these NLSs greatly facilitates HMGB1 enrichment in the cytoplasm in both immune cells and non-immune cells (Bonaldi et al., 2003; Andersson and Tracey, 2011). HMGB1 acetylation is regulated by both histone acetylase (HAT) and histone deacetylase (HDAC). HAT increases HMGB1 acetylation; whereas HDAC decreease HMGB1 acetylation (Topalova et al., 2008; Evankovich et al., 2010). During liver ischemia/reperfusion injury, the HDAC activity is significantly decreased, culminating in the hyperacetylation and nuclear translocation of HMGB1 (Evankovich et al., 2010). Consistently, HDAC knock-down by siRNA or pharmacological inhibition of HDAC activity by Trichostatin A drastically increased HMGB1 hyperacetylation, nuclear translocation, and release, in isolated hepatocytes (Evankovich et al., 2010). Although it is clear that immune cells release HMGB1 into the extracellular space through inflammasome-mediated pyroptosis, the mechanism of how immune cells regulate HMGB1 hyperacetylation and nuclear translocation in response to various infectious agents or endogenous danger signals remains largely unknown (Fig. 2); this clearly warrants investigation.

\section{ABBREVIATIONS}

AIM2, absent in melanoma 2; ASC, apoptosis-associated speck-like protein containing a CARD; HAT, histone acetylase; HDAC, histone deacetylase; HMGB1, high mobility group box 1; LRR, leucine-rich-repeat; MSU, monosodium uric; NLR, NOD-Like receptor; NLS, nuclear localization sequence; RAGE, receptor for advanced glycation end-

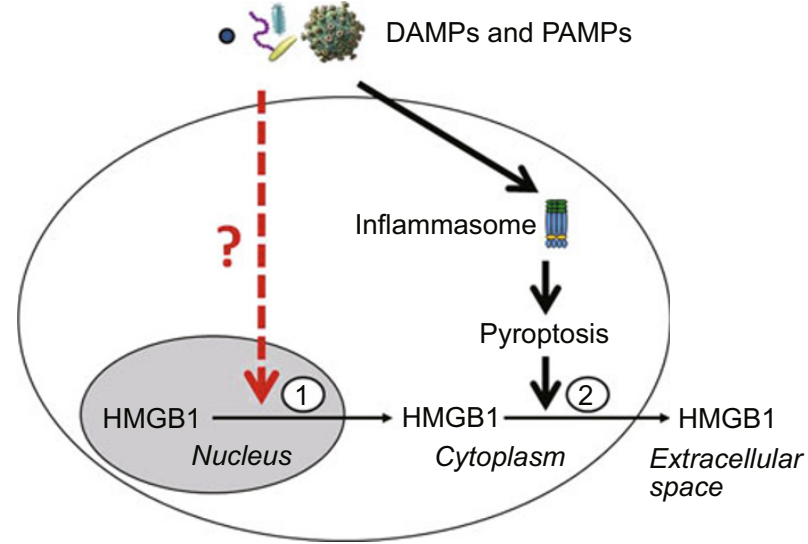

Figure 2. The mechanism of HMGB1 release from activated immune cells. There are two steps for HMGB1 release from activated immune cells. In the first step, DAMPs or PAMPs induce the translocation of HMGB1 from nucleus to cytoplasm via unknown mechanisms. In the second step, these DAMPs or PAMPs activate inflammasome, which in turn mediates HMGB1 release via pyroptosis.

products; TLR4, Toll-like receptor 4

\section{REFERENCES}

Andersson, U., and Tracey, K.J. (2011). HMGB1 is a therapeutic target for sterile inflammation and infection. Annu Rev Immunol 29, 139-162.

Bonaldi, T., Talamo, F., Scaffidi, P., Ferrera, D., Porto, A., Bachi, A., Rubartelli, A., Agresti, A., and Bianchi, M.E. (2003). Monocytic cells hyperacetylate chromatin protein HMGB1 to redirect it towards secretion. EMBO J 22, 5551-5560.

Bustin, M., and Neihart, N.K. (1979). Antibodies against chromosomal HMG proteins stain the cytoplasm of mammalian cells. Cell 16, 181-189.

Dey, M., Cao, C., Dar, A.C., Tamura, T., Ozato, K., Sicheri, F., and Dever, T.E. (2005). Mechanistic link between PKR dimerization, autophosphorylation, and elF2alpha substrate recognition. Cell 122, 901-913.

Evankovich, J., Cho, SW., Zhang, R., Cardinal, J., Dhupar, R., Zhang, L., Klune, J.R., Zlotnicki, J., Billiar, T., and Tsung, A. (2010). High mobility group box 1 release from hepatocytes during ischemia and reperfusion injury is mediated by decreased histone deacetylase activity. J Biol Chem. 285, 39888-39897.

Franchi, L., Muñoz-Planillo, R., and Núñez, G. (2012) Sensing and reacting to microbes through the inflammasomes. Nat Immunol 13, 325-332.

Gardella, S., Andrei, C., Ferrera, D., Lotti, L.V., Torrisi, M.R., Bianchi, M.E., and Rubartelli, A. (2002). The nuclear protein HMGB1 is secreted by monocytes via a non-classical, vesicle-mediated secretory pathway. EMBO Rep 3, 995-1001.

Hsu, L.C., Park, J.M., Zhang, K., Luo, J.L., Maeda, S., Kaufman, R.J., Eckmann, L., Guiney, D.G., and Karin, M. (2004). The protein kinase PKR is required for macrophage apoptosis after activation of Toll-like receptor 4. Nature 428, 341-345.

Javaherian, K., Liu, J.F., and Wang, J.C. (1978). Nonhistone proteins 
HMG1 and HMG2 change the DNA helical structure. Science 199, 1345-1346.

Jin, T., Perry, A., Jiang, J., Smith, P., Curry, J.A., Unterholzner, L., Jiang, Z., Horvath, G., Rathinam, V.A., and Johnstone, R.W., et al. (2012). Structures of the HIN domain:DNA complexes reveal ligand binding and activation mechanisms of the AIM2 inflammasome and IFI16 receptor. Immunity 36, 561-571.

Kayagaki, N., Warming, S., Lamkanfi, M., Vande Walle, L., Louie, S., Dong, J., Newton, K., Qu, Y., Liu, J., Heldens, S., el al. (2011). Noncanonical inflammasome activation targets caspase-11. Nature 479, 117-121.

Kofoed, E.M., and Vance, R.E. (2011). Innate immune recognition of bacterial ligands by NAIPs determines inflammasome specificity. Nature 477, 592-595.

Lamkanfi, M., Sarkar, A., Vande Walle, L., Vitari, A.C., Amer, A.O., Wewers, M.D., Tracey, K.J., Kanneganti, T.D., and Dixit, V.M. (2010). Inflammasome-dependent release of the alarmin HMGB1 in endotoxemia. J Immunol 185, 4385-4392.

Lu, B., Nakamura, T., Inouye, K., Li, J., Tang, Y., Lundbäck, P., ValdesFerrer, S.I., Olofsson, P.S., Kalb, T., and Roth, J., et al. (2012). Novel role of PKR in inflammasome activation and HMGB1 release. Nature 488, 670-674.

Manfredi, A.A., Capobianco, A., Esposito, A., De Cobelli, F., Canu, T., Monno, A., Raucci, A., Sanvito, F., Doglioni, C., Nawroth, P.P., et al. (2008). Maturing dendritic cells depend on RAGE for in vivo homing to lymph nodes. J Immunol 180, 2270-2275.

Maroso, M., Balosso, S., Ravizza, T., Liu, J., Aronica, E., Iyer, A.M., Rossetti, C., Molteni, M., Casalgrandi, M., Manfredi, A.A., et al. (2010). Toll-like receptor 4 and high-mobility group box-1 are involved in ictogenesis and can be targeted to reduce seizures. Nat Med 16, 413-419.

Medzhitov, R. (2010). Inflammation 2010: new adventures of an old flame. Cell 140, 771-776.

Miao, E.A., Rajan, J.V., and Aderem, A. (2011). Caspase-1-induced pyroptotic cell death. Immunol Rev 243, 206-214.

Müller, S., Scaffidi, P., Degryse, B., Bonaldi, T., Ronfani, L., Agresti, A., Beltrame, M., and Bianchi, M.E. (2001). New EMBO members' review: the double life of HMGB1 chromatin protein: architectural factor and extracellular signal. EMBO J 20, 4337-4340.

Nakamura, T., Furuhashi, M., Li, P., Cao, H., Tuncman, G., Sonenberg, N., Gorgun, C.Z., and Hotamisligil, G.S. (2010). Double-stranded RNA-dependent protein kinase links pathogen sensing with stress and metabolic homeostasis. Cell 140, 338-348.
Qin, S., Wang, H., Yuan, R., Li, H., Ochani, M., Ochani, K., RosasBallina, M., Czura, C.J., Huston, J.M., Miller, E., et al. (2006). Role of HMGB1 in apoptosis-mediated sepsis lethality. J Exp Med 203, 1637-1642.

Rathinam, V.A., Vanaja, S.K., and Fitzgerald, K.A. (2012). Regulation of inflammasome signaling. Nat Immunol 13, 333-332.

Scaffidi, P., Misteli, T., and Bianchi, M.E. (2002). Release of chromatin protein HMGB1 by necrotic cells triggers inflammation. Nature 418 , 191-195.

Schiraldi, M., Raucci, A., Muñoz, L.M., Livoti, E., Celona, B., Venereau, E., Apuzzo, T., De Marchis, F., Pedotti, M., Bachi, A., et al. (2012). HMGB1 promotes recruitment of inflammatory cells to damaged tissues by forming a complex with CXCL12 and signaling via CXCR4. J Exp Med 209, 551-563.

Strowig, T., Henao-Mejia, J., Elinav, E., and Flavell, R. (2012). Inflammasomes in health and disease. Nature 481, 278-286.

Wang, H., Bloom, O., Zhang, M., Vishnubhakat, J.M., Ombrellino, M., Che, J., Frazier, A., Yang, H., Ivanova, S., Borovikova, L., et al. (1999). HMG-1 as a late mediator of endotoxin lethality in mice. Science 285, 248-251.

Wen, H., Ting, J.P., and O'Neill, L.A. (2012). A role for the NLRP3 inflammasome in metabolic diseases--did Warburg miss inflammation. Nat Immunol 13, 352-357.

Willingham, S.B., Allen, I.C., Bergstralh, D.T., Brickey, W.J., Huang, M.T., Taxman, D.J., Duncan, J.A., and Ting, J.P. (2009). NLRP3 (NALP3, Cryopyrin) facilitates in vivo caspase-1 activation, necrosis, and HMGB1 release via inflammasome-dependent and -independent pathways. J Immunol 182, 6460-6469.

Topalova, D., Ugrinova, I., Pashev, I.G., and Pasheva, E.A. (2008). HMGB1 protein inhibits DNA replication in vitro: a role of the acetylation and the acidic tail. Int J Biochem Cell Biol 40, 1536-1542.

Venereau, E., Casalgrandi, M., Schiraldi, M., Antoine, D.J., Cattaneo, A., De Marchis, F., Liu, J., Antonelli, A., Preti, A., Raeli, L., et al. (2012). Mutually exclusive redox forms of HMGB1 promote cell recruitment or proinflammatory cytokine release. J Exp Med 209, 1519-1528.

Yang, H., Hreggvidsdottir, H.S., Palmblad, K., Wang, H., Ochani, M., Li, J., Lu, B., Chavan, S., Rosas-Ballina, M., Al-Abed, Y., et al. (2010). A critical cysteine is required for HMGB1 binding to Toll-like receptor 4 and activation of macrophage cytokine release. Proc Natl Acad Sci U S A 107, 11942-11947.

Zhao, Y., Yang, J., Shi, J., Gong, Y.N., Lu, Q., Xu, H., Liu, L., and Shao, F. (2011). The NLRC4 inflammasome receptors for bacterial flagellin and type III secretion apparatus. Nature 477, 596-600. 